\title{
Hybrid Berezinskii-Kosterlitz-Thouless and Ising topological phase transition in the generalized two-dimensional XY model using tensor networks
}

\author{
Feng-Feng Song ${ }^{1}$ and Guang-Ming Zhang ${ }^{1,2}$ \\ ${ }^{1}$ State Key Laboratory of Low-Dimensional Quantum Physics and \\ Department of Physics, Tsinghua University, Beijing 100084, China. \\ ${ }^{2}$ Frontier Science Center for Quantum Information, Beijing 100084, China.
}

(Dated: January 6, 2021)

\begin{abstract}
In tensor network representation, the partition function of a generalized two-dimensional XY spin model with topological integer and half-integer vortex excitations is mapped to a tensor product of one-dimensional quantum transfer operator, whose eigen-equation can be solved by an algorithm of variational uniform matrix product states. Using the singularities of the entanglement entropy, we accurately determine the complete phase diagram of this model. Both the integer vortex-antivortex binding and half-integer vortex-antivortex binding phases are separated from the disordered phase by the usual Berezinskii-Kosterlitz-Thouless (BKT) transitions, while a continuous topological phase transition exists between two different vortex binding phases, exhibiting a logarithmic divergence of the specific heat and exponential divergence of the spin correlation length. A new hybrid BKT and Ising universality class of topological phase transition is thus established. We further prove that three phase transition lines meet at a multi-critical point, from which a deconfinement crossover line extends into the disordered phase.
\end{abstract}

Introduction. -It is well-known that topological vortices/defects govern the critical behavior and produce rich physics in two dimensional (2D) systems. One prominent example is the Berezinskii-Kosterlitz-Thouless (BKT) phase transition [1, 2, which is associated with the binding of integer vortices and antivortices in pairs with quasi-long-range order. Such a transition cannot be characterized by spontaneous symmetry breaking with local order parameter and constitutes the first example of topological phase transitions beyond the Landau paradigm. A prototype model exhibiting these fascinating features is given by the 2D classical XY spin model, which can be realized in superfluid helium films [3] and $2 \mathrm{D}$ superconductor films/arrays [4] 6 . When an extra spin-nematic interaction with $\pi$ period is introduced, the generalized XY model contains both integer vortices and half-integer vortices along with their associated topological strings 7 , 8. Although great efforts have been devoted to establish a complete phase diagram 91 13, there are still some standing puzzles: what is the nature of the phase transition between the integer vortex binding and half-integer vortex binding phases [8, 10], how does this transition line merge into two BKT transition lines[11] and is there a deconfinement transition in the disordered phase [12]? The accurate determination of the transition lines and the possible multi-critical point remain a great challenge.

Recently, tensor network method has become a powerful theoretical tool to characterize correlated quantum many-body phases in the thermodynamic limit [14, 15]. Since the partition function of a $2 \mathrm{D}$ classical statistical model can be represented as a tensor product of $1 \mathrm{D}$ quantum transfer operator [16, the eigen-equation of this transfer operator can be solved by the variational uniform matrix product state (VUMPS) algorithm[17-19, and all thermodynamic properties are thus accurately obtained. In this scheme, the critical temperature of the BKT transition in the usual 2D XY model is estimated with high precision and the exponential divergence of the spin correlation length is confirmed 20]. Therefore, it becomes possible to apply the tensor network approach to resolve those puzzles about the generalized XY spin model, shedding new light on the nature of the topological phase transition between two different vortex binding phases.

In this paper, we carry out such tensor network calculations for the generalized 2D XY spin model, and accurately determine the topological phase transitions using the singularity of the entanglement entropy associated to the 1D quantum transfer operator. Thus the complete phase diagram of the generalized XY model is precisely obtained. In particular, we show that a continuous phase transition occurs between two different vortex binding phases, where the logarithmic divergence of the specific heat of the 2D Ising transition and the exponential divergence of the spin correlation length of the BKT transition simultaneously exist, establishing a new hybrid BKT and Ising universality class with two different correlation lengths. From the analysis of the entanglement spectrum, a deconfinement crossover line is found in the disordered phase, separating the region with dominant integer vortex excitations from the region with dominant half-integer vortex excitations.

Model and Method. -The generalized XY spin model on a $2 \mathrm{D}$ square lattice is given by

$$
H=-J \sum_{\langle i j\rangle}\left[\Delta \cos \left(\theta_{i}-\theta_{j}\right)+(1-\Delta) \cos \left(2 \theta_{i}-2 \theta_{j}\right)\right]
$$

where $\theta_{i} \in[0,2 \pi]$ denotes the spin orientation at the lattice site $i$ and the summation runs over all nearest neighbor sites. $\Delta=1$ corresponds to the usual XY spin model 
with integer vortex excitations, while $\Delta=0$ corresponds to a purely spin-nematic model with the invariance un$\operatorname{der} \theta_{i} \rightarrow \theta_{i}+\pi$. In the latter, the main topological excitations are half-integer vortices and their associated strings across which spins are antiparallel, and the socalled half-BKT transition occurs at the exactly same critical temperature as the usual XY model, characterized by the binding of half-integer vortices and antivortices in pairs at low temperatures. For $0<\Delta<1$, two local interactions strongly compete with each other, and the system undergoes a phase transition from the integer vortex-antivortex binding phase to the half-integer vortex-antivortex binding phase 8 .

The tensor network method starts from expressing the partition function as a tensor network

$$
Z=\prod_{i} \int \frac{\mathrm{d} \theta_{i}}{2 \pi} \prod_{\langle i j\rangle} \mathrm{e}^{\beta\left[\Delta \cos \left(\theta_{i}-\theta_{j}\right)+(1-\Delta) \cos 2\left(\theta_{i}-\theta_{j}\right)\right]},
$$

where the temperature $T$ is in the unit $J / k_{B}$. To find its tensor network representation, we take a duality transformation, which changes the phase variables into number indices on the links. Such a transformation is obtained by the character expansion for the Boltzmann factor $e^{x \cos \theta}=\sum_{n=-\infty}^{\infty} I_{n}(x) e^{i n \theta}$, where $I_{n}(x)$ are the modified Bessel functions of the first kind. Then the partition function is written as

$$
Z=\prod_{s} \int \frac{\mathrm{d} \theta_{s}}{2 \pi} \prod_{l \in \mathcal{L}} \sum_{n_{l}} a_{n_{l}}(\beta, \Delta) \mathrm{e}^{i n_{l}\left(\theta_{s_{i}}-\theta_{s_{j}}\right)},
$$

where $a_{n}(\beta, \Delta)=\sum_{m=-\infty}^{+\infty} I_{n-2 m}(\beta \Delta) I_{m}(\beta(1-\Delta)), l$ runs over all the links and $s$ labels all the lattice sites. By integrating out the physical degrees of freedom $\theta$, the partition function is represented as a tensor network

$$
Z=\operatorname{tTr} \prod_{s} O_{n_{1}, n_{2}}^{n_{3}, n_{4}}(s),
$$

where tTr denotes the tensor contraction and each local tensor is given by $O_{n_{1}, n_{2}}^{n_{3}, n_{4}}=\left(\prod_{i=1}^{4} a_{n_{i}}(\beta, \Delta)\right)^{1 / 2} \delta_{n_{1}+n_{2}}^{n_{3}+n_{4}}$. The partition function is shown in Fig. 1 (a). The continuous symmetry of the model has been encoded in the tensor network representation as the constraint of vanishing lattice divergence: $O_{n_{1}, n_{2}}^{n_{3}, n_{4}} \neq 0$ only if $n_{1}+n_{2}-n_{3}-n_{4}=$ 0 , i.e., the conservation law of $U(1)$ charges. Since the expansion coefficients decrease exponentially with increasing $n$, an appropriate truncation can be performed on the virtual legs of the tensor $O$ without loss of accuracy. Actually, the dual index $n$ plays the essential role in describing the Ising physics when interpreted as the curl of the height variable $h_{a}$ on the dual lattice, where the corresponding dual Ising spin is defined by [12] $\mu_{a}=(-1)^{h_{a}}$.

The fundamental object in the tensor-network partition function is the row-to-row quantum transfer operator,

$$
\widehat{T}(\beta, \Delta)=\sum_{\ldots s, p, q \ldots} \operatorname{Tr}\left(\cdots O_{n_{1}}^{n_{3}}(s) O_{n_{1}}^{n_{3}}(p) O_{n_{1}}^{n_{3}}(q) \cdots\right),
$$

(a)

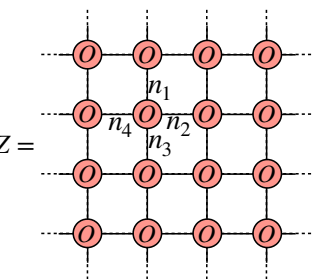

(c)

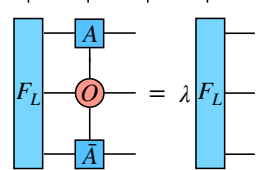

(b)

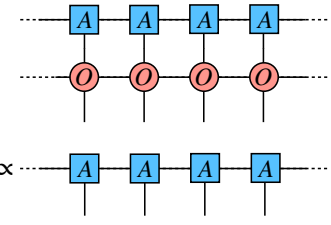

(d)

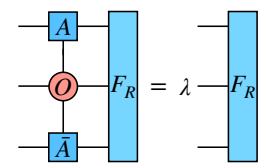

(e)

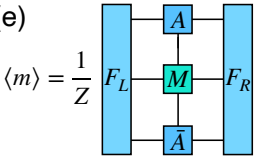

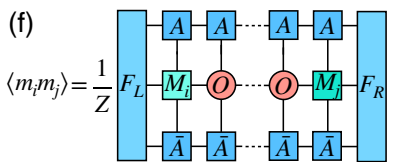

FIG. 1: (a) Tensor network representation of the partition function. (b) Eigen-equation for the fixed-point uMPS of the transfer operator $T(\beta, \Delta)$. (c) and (d) Eigen-equations for the left and right fixed-point eigenvectors of the channel operator $\mathbb{T}_{O}$. (e) Expectation of a local operator by contracting the leading eigenvectors of the channel operators. (f) Two-point correlation function represented by contracting a sequence of channel operators.

where $s, p, q \ldots$ refer to the lattice sites in a row. This operator plays the same role as the matrix product operator (MPO) for 1D quantum spin chains [16, whose model Hamiltonian can be formally expressed as

$$
\hat{H}_{1 d}=-\frac{1}{\beta} \log \widehat{T}(\beta, \Delta) .
$$

Then the partition function is determined by the dominant eigenvalues of $\widehat{T}(\beta, \Delta)$. For a translation-invariant $\mathrm{MPO}$, the leading eigenvector as the fixed-point of $\widehat{T}(\beta, \Delta)$ can be represented by a uniform matrix product states (uMPS) 17

$$
|\Psi(A)\rangle=\sum_{\ldots s, p, q \ldots} \operatorname{Tr}\left(\cdots A^{n_{s}} A^{n_{p}} A^{n_{q}} \cdots\right),
$$

where $A_{\alpha \beta}^{n_{s}}$ is a three-leg tensor with bond dimension $D$, controlling the accuracy of this approximation. Since the transfer operator $\hat{T}(\beta, \Delta)$ is Hermitian, the eigenequation as shown in Fig. 1 (b)

$$
\widehat{T}(\beta, \Delta)|\Psi(A)\rangle=\Lambda_{\max }|\Psi(A)\rangle,
$$

can be transformed as an optimization problem

$$
\max _{A}\langle\Psi(A)|\widehat{T}(\beta, \Delta)| \Psi(A)\rangle /\langle\Psi(A) \mid \Psi(A)\rangle,
$$

for the local tensor $A$. To solve this optimization problem, we apply the VUMPS algorithm[17-19], which provides an efficient variational scheme to approximate the largest eigenvector $|\Psi(A)\rangle$. Then various physical quantities can be estimated from the fixed-point uMPS. 
For the entanglement entropy, we perform a bipartition on $|\Psi(A)\rangle$ via Schmidt decomposition

$$
|\Psi(A)\rangle=\sum_{\alpha, \beta=1}^{D} s_{\alpha} \delta_{\alpha, \beta}\left|\Psi_{\alpha}^{-\infty, n}\right\rangle\left|\Psi_{\beta}^{n+1, \infty}\right\rangle .
$$

where $s_{\alpha}$ are the singular values. The entanglement entropy $S_{E}$ is given by

$$
S_{E}=-\sum_{\alpha=1}^{D} s_{\alpha}^{2} \ln s_{\alpha}^{2}
$$

in the same way as the quantum entanglement measure for a many-body quantum state 21. Moreover, the entanglement spectrum 22 can be defined by $\varepsilon_{n}=-\log s_{n}^{2}$ to yield more information on the fixed-point uMPS.

Moreover, the evaluations of a local observable $m\left(\theta_{i}\right)$ can be represented as

$$
\left\langle m\left(\theta_{i}\right)\right\rangle=\frac{1}{Z} \prod_{j} \int \frac{\mathrm{d} \theta_{j}}{2 \pi} \mathrm{e}^{-\beta E\left(\left\{\theta_{j}\right\}\right)} m\left(\theta_{i}\right),
$$

where $E\left(\left\{\theta_{j}\right\}\right)$ is the energy under a given configuration $\left\{\theta_{j}\right\}$. Compared to the partition function (2), the $m\left(\theta_{i}\right)$ on the right hand side of Eq. 10 simply changes the tensor $O_{n_{1}, n_{2}}^{n_{3}, n_{4}}(i)$ into an impurity local tensor

$M_{n_{1}, n_{2}}^{n_{3}, n_{4}}=\left(\prod_{i=1}^{4} a_{n_{i}}(\beta, \Delta)\right)^{1 / 2} \int \frac{\mathrm{d} \theta}{2 \pi} \mathrm{e}^{i \theta\left(n_{1}+n_{2}-n_{3}-n_{4}\right)} m(\theta)$.

Using the uMPS fixed-point, the contraction of the tensor network of $\langle m(\theta)\rangle$ is reduced to a trace of an infinite sequence of channel operators

$$
\langle m(\theta)\rangle=\operatorname{Tr}\left(\cdots \mathbb{T}_{O} \mathbb{T}_{O} \mathbb{T}_{M} \mathbb{T}_{O} \mathbb{T}_{O} \cdots\right),
$$

where the channel operator is defined by

$$
\mathbb{T}_{X}=\sum_{i, j} \bar{A}^{i} \otimes X^{i, j} \otimes A^{j}
$$

In the same fashion, the contraction of channel operators is determined by the leading eigenvectors $\left\langle F_{L}\right|$ and $\left|F_{R}\right\rangle$ of $\mathbb{T}_{O}$ as shown in Fig. 1 (c) and (d). Thus the expectation value of a local observable can be obtained by contraction of the leading eigenvectors $\left\langle F_{L}\right|$ and $\left|F_{R}\right\rangle$ with $\mathbb{T}_{M}$,

$$
\langle m(\theta)\rangle=\left\langle F_{L}\left|\mathbb{T}_{M}\right| F_{R}\right\rangle,
$$

as shown in Fig. 1 (e). Moreover, the correlation functions between two local observable $m\left(\theta_{i}\right)$ and $m\left(\theta_{j}\right)$ defined by $G(r)=\left\langle m\left(\theta_{i}\right) m\left(\theta_{j}\right)\right\rangle$ can also be represented as a contraction of a tensor network with two local impurity tensors $M_{i}$ and $M_{j}$. As displayed in Fig. 1 (f), with the
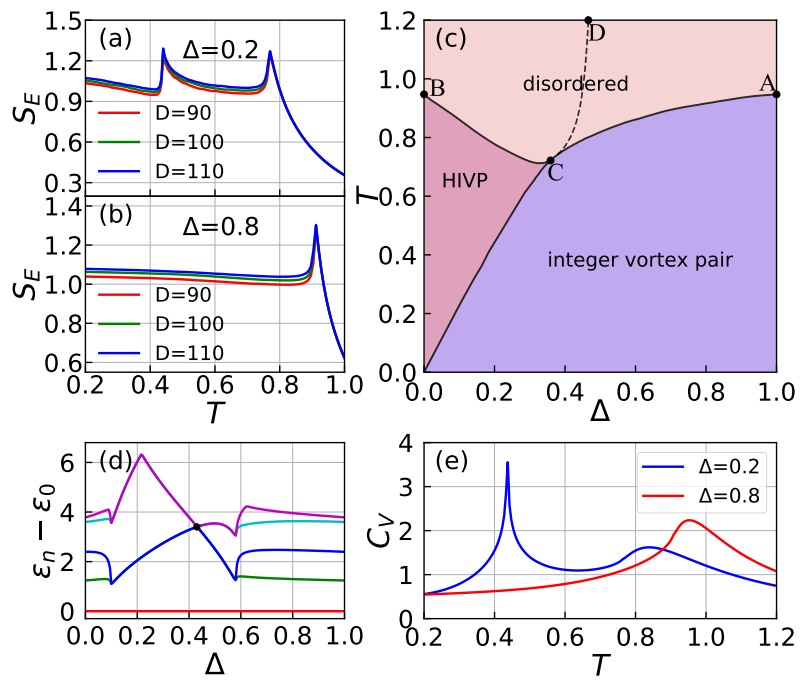

FIG. 2: (a) and (b) The entanglement entropy as a function of temperature for $\Delta=0.2$ and $\Delta=0.8$. (c) The complete phase diagram of the generalized $2 \mathrm{D} \mathrm{XY}$ model. $A C$ and $B C$ are two BKT transition lines, and there is a special continuous transition line from the origin to a multi-critical point $\mathrm{C}$. HIVP is an abbreviation for the half-integer vortex pair. (d) The five lowest entanglement levels of the fixed-point uMPS under $T=0.85$. There is a four-fold degenerate crossing point (black dot) corresponding to the deconfinement crossover in the disordered phase. (e) The specific heat as a function of temperature for $\Delta=0.2$ and $\Delta=0.8$.

help of fixed-points of the channel operator, we can easily derive

$$
G(r)=\left\langle F_{L}|\mathbb{T}_{M_{i}} \underbrace{\mathbb{T}_{O} \cdots \mathbb{T}_{O}}_{r-1} \mathbb{T}_{M_{j}}| F_{R}\right\rangle .
$$

Numerical results. -In the tensor network framework, the entanglement entropy of the fixed-point uMPS for the 1D quantum transfer operator exhibits singularity, which can be used to accurately determine all possible phase transitions. First, we estimate the critical temperature of the BKT transition for the usual XY model $(\Delta=1)$ as $T_{\mathrm{BKT}} \simeq 0.8933$, sufficiently close to the result from high-temperature expansion with high precision 23,24 . Then we calculate the entanglement entropy for a general value of $0<\Delta<1$. In Fig. 2(a) and (b), the entanglement entropy $S_{E}$ develops two sharp peaks at $T_{c, 1} \simeq 0.44$ and $T_{c, 2} \simeq 0.77$ for a small value $\Delta=0.2$, and one sharp peak at $T_{c} \simeq 0.91$ for a large value $\Delta=0.8$. The peak positions are almost unchanged with the bond dimensions $D=90,100,110$. So we can accurately locate the phase boundaries, and the complete phase diagram is thus derived as displayed in Fig. 2(c). We find two BKT phase transition lines $A C$ and $B C$ corresponding to the integer vortex-antivortex binding and the half-integer vortex-antivortex binding transitions, respectively. More importantly, there is another topological phase transition line between the integer vortex-antivortex binding phase 
and the half-integer vortex-antivortex binding phase, and three phase transition lines merge at a multi-critical point $C(\Delta \simeq 0.330, T \simeq 0.705)$.

Furthermore the entanglement spectrum of the fixedpoint uMPS for the 1D quantum transfer operator exhibits some intriguing features. In Fig. 2 (d), the five lowest entanglement levels are displayed for a given temperature $T=0.85$, where varying $\Delta$ goes through all three phases. The lowest entanglement level is always non-degenerate. Exactly at the two critical BKT transitions $\Delta_{c, 1} \simeq 0.10$ and $\Delta_{c, 2} \simeq 0.58$, the first and second excited entanglement levels become degenerate when entering the disordered phase. Inside the disordered phase, we find an unusual feature that the two-fold degenerate excited levels have a further crossing to form a four-fold degenerate point at $\Delta^{*} \simeq 0.43$. With a careful numerical analysis, we find that this four-fold degenerate point just starts from the multi-critical point $C$ and extends into the disordered phase. In the phase diagram Fig. 2 (c), these highly degenerate points are plotted in a dotted line, which approaches $\Delta=0.5$ asymptotically as increasing the temperature. According to the discussion from the dual height model[12, we identify this special line as the deconfinement crossover line separating the region with dominant integer vortex excitations $(0.5<\Delta<1)$ from the half-integer vortex dominant region $(0<\Delta<0.5)$.

To further understand the topological phase transition between two different vortex binding phases, we calculate the specific heat, which can be represented in the tensor-network language based on two nearest neighbor impurity tensors. Using the contraction in (14), the internal energy per site can be calculated as

$$
u=-\Delta\left\langle\cos \left(\theta_{i}-\theta_{i+1}\right)\right\rangle-(1-\Delta)\left\langle\cos \left(2 \theta_{i}-2 \theta_{i+1}\right)\right\rangle,
$$

and the specific heat per site is given by $C_{V}=\partial u / \partial T$. For $\Delta=0.2$, all three phases can be reached as varying the temperature, while for $\Delta=0.8$ there are only two phases. As shown in Fig. 2(e), the specific heat shows a bump around two BKT type transitions but a logarithmic divergence at the transition between two different vortex binding phases. Such a logarithmic specific heat is usually regarded as a characteristic feature of the $2 \mathrm{D}$ Ising transition 7 12.

Actually the nature of this phase transition between two different vortex binding phases can also be revealed by studying the following correlation functions

$$
G_{1}(r)=\left\langle\cos \left(\theta_{i}-\theta_{i+r}\right)\right\rangle, \quad G_{2}(r)=\left\langle\cos \left(2 \theta_{i}-2 \theta_{i+r}\right)\right\rangle,
$$

which describe the correlations of the spins and nematic spins, respectively. Within the tensor network framework, these two correlation functions can be calculated readily using local impurity tensors of $e^{ \pm i \theta_{i}}$ and $e^{ \pm i 2 \theta_{i}}$. It has been known that $G_{1}(r)$ decays algebraically only in the integer vortex-antivortex binding phase, while $G_{2}(r)$ exhibits algebraic decay both in the half-integer vortexantivortex binding and integer vortex-antivortex binding
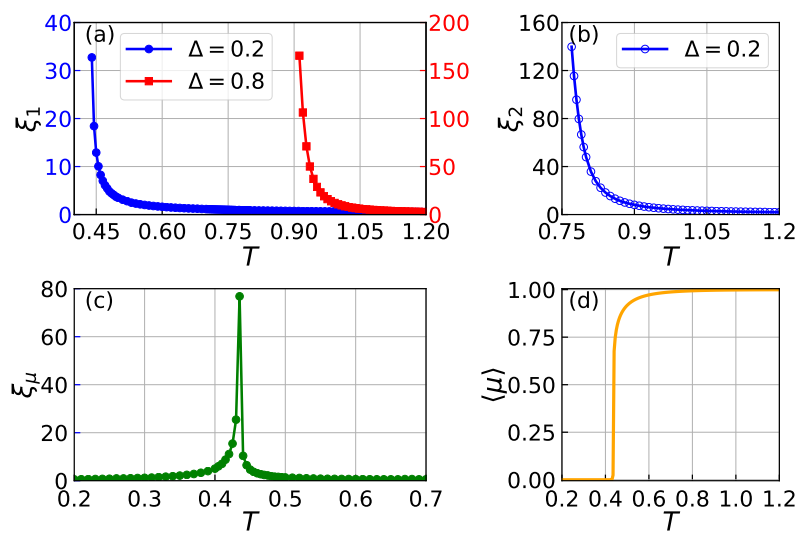

FIG. 3: (a) and (b) Correlation lengths derived from the spin and nematic spin correlation functions with bond dimension $D=110$. (c) The dual Ising correlation length extracted from the dual Ising spin correlator along $\Delta=0.2$. (d) Expectation value of the dual Ising spins along the line $\Delta=0.2$ in the phase diagram.

phases $\left[8,9\right.$. When $G_{1}(r)$ and $G_{2}(r)$ decay exponentially, two different characteristic lengths $\xi_{1}$ and $\xi_{2}$ are defined by 13

$$
G_{1}(r) \sim \exp \left(-r / \xi_{1}\right), \quad G_{2}(r) \sim \exp \left(-r / \xi_{2}\right) .
$$

In particular, $G_{1}(r)$ decays exponentially in the halfinteger vortex-antivortex binding phase, so we can study the critical behavior of the correlation length $\xi_{1}$ when approaching the phase transition. In Fig. 3 (a), we show the correlation length $\xi_{1}$ as a function of temperature for $\Delta=0.2$ with a sharp divergence above the critical point $T_{c} \simeq 0.44$, as well as $\Delta=0.8$ with a sharp divergence above the BKT transition point $T_{c} \simeq 0.91$. Similarly, the correlation length $\xi_{2}$ for $\Delta=0.2$ as displayed in Fig. 3 (b) has a divergence above the half-BKT transition point $T_{c} \simeq 0.77$. When approaching the critical points from the high-temperature side, all correlation lengths are found to be well-fitted by an exponentially divergent form

$$
\xi(T) \propto \exp \left(\frac{b}{\sqrt{T-T_{C}}}\right), T \rightarrow T_{C}^{+}
$$

where $b$ is a non-universal positive constant, which is a characteristic property of the BKT transition 25].

Moreover, the two-point correlator characterizing the Ising strings is defined by

$$
\begin{aligned}
C(r) & =\langle\mu(i) \mu(i+r)\rangle \\
& =\left\langle(-1)^{n_{i}}(-1)^{n_{i+1}} \cdots(-1)^{n_{i+r-1}}\right\rangle .
\end{aligned}
$$

So the correlation function of the dual Ising spin variables is transformed into an expectation of a string of impurity variables $(-1)^{n_{i}}$. On both sides of the critical point $T_{c} \simeq 0.44$, we find $C(r)$ decays exponentially, and the corresponding Ising correlation length $\xi_{\mu}$ is extracted 


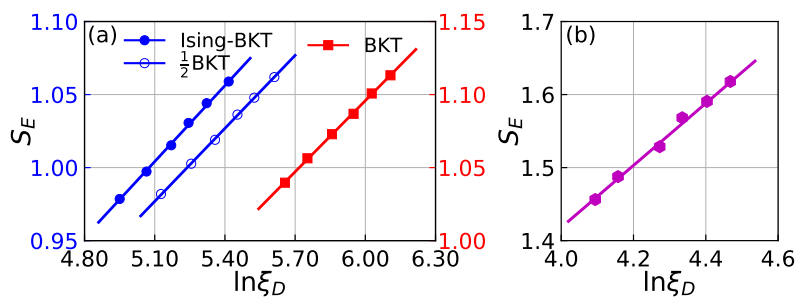

FIG. 4: (a) Central charges extracted from a linear fit of the entanglement entropy with increasing the bond dimensions from 110 to 160: $c \simeq 0.992$ for the Ising-BKT transition, $c \simeq 0.999$ for the BKT transition, and $c \simeq 0.996$ for the halfBKT transition. (b) The central charge $c \simeq 2.492$ is extracted from the correlation length close to the multi-critical point $C$.

and displayed in Fig. 3 (c). When approaching $T_{c}$ from either side, $\xi_{\mu}$ diverges as $1 /\left|T-T_{c}\right|$ in the same way of the 2D Ising transition. In Fig. 3(d) we also show the dual Ising order parameter and $\langle\mu\rangle>0$ indicates the proliferation of Ising domain walls. All these results strongly suggest that the transition from the half-integer vortexantivortex binding phase to the integer vortex-antivortex binding phase is a hybrid BKT and Ising transition. Two different correlation lengths are needed to characterize this new universality class of topological phase transition.

Furthermore, we can extract the central charges for those critical field theories by numerically fitting the entanglement entropy with the correlation lengths obtained under different bond dimensions [26]

$$
S_{E} \propto \frac{c}{6} \ln \xi_{D},
$$

where $\xi_{D}$ is the largest correlation length with the different bond dimensions. In Fig. 4 (a), the numerical fitting clearly shows the central charges equal to $c=1$ for all three transitions, precisely the value of the BKT-type transition. More importantly, close to the multi-critical point $C$, we can also deduce the leading correlation length from the fixed-point uMPS from the disorder phase. In Fig. 4 (b), by fitting the correlation lengths determines the corresponding central charge as $c \simeq 5 / 2$. There is a possible way to explain this result. Since two different correlation lengths are involved in the Ising-BKT transition, the corresponding critical field theory should be characterized by a $2 \mathrm{D}$ conformal field theory with a central charge $c=1+1 / 2$, and this Ising-BKT transition line further merges with the BKT transition line $A C B$ at the multi-critical point $C$, leading to the central charge $c=1+3 / 2$.

Conclusion and Outlook. -We have employed the tensor network method to solve the generalized twodimensional classical XY spin model with topological integer and half-integer vortex excitations as well as the string excitations. Using the singularity of the entanglement entropy, we have accurately determined the phase diagram of this model. In particular, a new hybrid BKT and Ising phase transition has been established between the integer vortex-antivortex binding phase and the halfinteger vortex-antivortex binding phase. To describe this new phase transition, two distinctly characteristic correlation lengths are necessary, the corresponding critical field theory may be characterized by a $2 \mathrm{D}$ conformal field theory with the $Z_{2} \times U(1)$ symmetry.

In recent years, a large amount of investigations have focused on a gas of attractive bosons, which can form two distinct superfluid phases: an atomic superfluid of bosons and a molecular superfluid of boson pairs 27,29 . The former corresponds to the integer vortex-antivortex binding phase and the latter is the half-integer vortexantivortex binding phase. The established hybrid topological phase transition may be experimentally realized in these physical systems.

\section{Acknowledgments}

The research is supported by the National Key Research and Development Program of MOST of China (2017YFA0302902).

[1] V. Berezinsky, Sov. Phys. JETP 32, 493 (1971).

[2] J. M. Kosterlitz and D. J. Thouless, Journal of Physics C: Solid State Physics 6, 1181 (1973), URL https:// doi.org/ $10.1088 \% 2 \mathrm{~F} 0022-3719 \% 2 \mathrm{~F} 6 \% 2 \mathrm{~F} 7 \% 2 \mathrm{~F} 010$.

[3] D. J. Bishop and J. D. Reppy, Phys. Rev. Lett. 40, 1727 (1978), URL https://link.aps.org/doi/10. 1103/PhysRevLett.40.1727.

[4] A. F. Hebard and A. T. Fiory, Phys. Rev. Lett. 44, 291 (1980), URL https://link.aps.org/doi/10.1103/ PhysRevLett.44.291.

[5] K. Epstein, A. M. Goldman, and A. M. Kadin, Phys. Rev. Lett. 47, 534 (1981), URL https://link.aps.org/doi/ 10.1103/PhysRevLett.47.534

[6] D. J. Resnick, J. C. Garland, J. T. Boyd, S. Shoemaker, and R. S. Newrock, Phys. Rev. Lett. 47, 1542 (1981), URL https://link.aps.org/doi/10. 1103/PhysRevLett.47.1542.

[7] S. Korshunov, JETP Lett 41 (1985).

[8] D. H. Lee and G. Grinstein, Phys. Rev. Lett. 55, 541 (1985), URL https://link.aps.org/doi/10.1103/ PhysRevLett.55.541.

[9] D. B. Carpenter and J. T. Chalker, Journal of Physics: Condensed Matter 1, 4907 (1989), URL https://doi. org/ $10.1088 \% 2 \mathrm{~F} 0953-8984 \% 2 \mathrm{~F} 1 \% 2 \mathrm{~F} 30 \% 2 \mathrm{~F} 004$.

[10] Y. Shi, A. Lamacraft, and P. Fendley, Phys. Rev. Lett. 107, 240601 (2011), URL https://link.aps.org/doi/ 10.1103/PhysRevLett.107.240601.

[11] D. M. Hübscher and S. Wessel, Phys. Rev. E 87, 062112 (2013), URL https://link.aps.org/doi/10. 1103/PhysRevE.87.062112

[12] P. Serna, J. T. Chalker, and P. Fendley, Journal of Physics A: Mathematical and Theoretical 50, 424003 (2017), URL https://doi.org/10.1088\%2F1751-8121\% 2Faa89a1

[13] D. X. Nui, L. Tuan, N. D. Trung Kien, P. T. Huy, 
H. T. Dang, and D. X. Viet, Phys. Rev. B 98, 144421 (2018), URL https://link.aps.org/doi/10. 1103/PhysRevB.98.144421

[14] F. Verstraete, V. Murg, and J. Cirac, Advances in Physics 57, 143 (2008), https://doi.org/10.1080/14789940801912366, URL https://doi.org/10.1080/14789940801912366.

[15] R. Orús, Annals of Physics 349, 117 (2014), ISSN 00034916, URL http://www.sciencedirect.com/science/ article/pii/S0003491614001596.

[16] J. Haegeman and F. Verstraete, Annual Review of Condensed Matter Physics 8, 355 (2017), https://doi.org/10.1146/annurev-conmatphys031016-025507, URL https://doi.org/10.1146/ annurev-conmatphys-031016-025507

[17] V. Zauner-Stauber, L. Vanderstraeten, M. T. Fishman, F. Verstraete, and J. Haegeman, Phys. Rev. B 97, 045145 (2018), URL https://link.aps.org/doi/ 10.1103/PhysRevB.97.045145.

[18] M. T. Fishman, L. Vanderstraeten, V. Zauner-Stauber, J. Haegeman, and F. Verstraete, Phys. Rev. B 98, 235148 (2018), URL https://link.aps.org/doi/10. 1103/PhysRevB.98.235148

[19] L. Vanderstraeten, J. Haegeman, and F. Verstraete, SciPost Phys. Lect. Notes p. 7 (2019), URL https: //scipost.org/10.21468/SciPostPhysLectNotes.7

[20] L. Vanderstraeten, B. Vanhecke, A. M. Läuchli, and F. Verstraete, Phys. Rev. E 100, 062136 (2019), URL https://link.aps.org/doi/10.1103/PhysRevE. 100.062136
[21] G. Vidal, J. I. Latorre, E. Rico, and A. Kitaev, Phys. Rev. Lett. 90, 227902 (2003), URL https://link.aps. org/doi/10.1103/PhysRevLett.90.227902.

[22] H. Li and F. D. M. Haldane, Phys. Rev. Lett. 101, 010504 (2008), URL https://link.aps.org/doi/10. 1103/PhysRevLett.101.010504

[23] H. Arisue, Phys. Rev. E 79, 011107 (2009), URL https: //link.aps.org/doi/10.1103/PhysRevE.79.011107.

[24] Y.-D. Hsieh, Y.-J. Kao, and A. W. Sandvik, Journal of Statistical Mechanics: Theory and Experiment 2013, P09001 (2013), URL https://doi.org/10.1088\% 2F1742-5468\%2F2013\%2F09\%2Fp09001.

[25] J. M. Kosterlitz, Journal of Physics C: Solid State Physics 7, 1046 (1974), URL https://doi.org/10. 1088\%2F0022-3719\%2F7\%2F6\%2F005

[26] F. Pollmann, S. Mukerjee, A. M. Turner, and J. E. Moore, Phys. Rev. Lett. 102, 255701 (2009), URL https://link.aps.org/doi/10.1103/PhysRevLett. 102.255701.

[27] M. W. J. Romans, R. A. Duine, S. Sachdev, and H. T. C. Stoof, Phys. Rev. Lett. 93, 020405 (2004), URL https: // link.aps.org/doi/10.1103/PhysRevLett.93.020405.

[28] L. Radzihovsky, J. Park, and P. B. Weichman, Phys. Rev. Lett. 92, 160402 (2004), URL https://link.aps.org/ doi/10.1103/PhysRevLett.92.160402

[29] L. Bonnes and S. Wessel, Phys. Rev. B 85, 094513 (2012), URL https://link.aps.org/doi/10. 1103/PhysRevB.85.094513 